\title{
La actividad física: entre la ética y la estética. Una aproximación a la luz de la concepción hipocrática
}

Pablo Zanor ${ }^{1}$

Universidad Nacional de la Matanza

\section{Ensayo}

Material original autorizado para su primera publicación en el Journal de Ciencias Sociales, Revista Académica de la Facultad de Ciencias Sociales de la Universidad de Palermo.

Recepción: 7-09-2021

Aceptación: 27-09-2021

Resumen: El siguiente ensayo tiene por finalidad proponer la concepción hipocrática de la actividad física como paradigma o al menos como una orientación de la actividad física actual. El texto comienza contextualizando la actividad física hipocrática dentro del marco de la Paideia griega, es decir, dentro de una sociedad orgánica que buscaba a partir del areté una educación integral de la persona. La segunda parte se interroga sobre el significado que tiene hoy la actividad física dentro de una sociedad compleja. Aquí señalaremos la reducción de la actividad física a su dimensión estética asociada a un cuerpo bello. Finalmente, intentaremos proponer la importancia de volver a vivir la actividad física desde la óptica hipocrática para devolverle su misión educadora. Esto no significa soslayar las dimensiones estéticas que también son parte de la actividad física.

Palabras clave: actividad física, teoría hipocrática, tiranía de la belleza, identidad de la persona.

\section{Physical activity: between ethics and aesthetics. An approach from the Hippocratic conception}

Abstract: The following essay aims to propose the Hippocratic conception of physical activity as a paradigm or at least as an orientation of current physical activity. The text begins by contextualizing the Hippocratic physical activity within the framework of the Greek Paideia, that is, within an organic society that sought from the areté an integral education of the person. The second part asks about the meaning that physical activity has today in a complex society. Here we will point out the reduction of physical activity to its aesthetic dimension associated

\footnotetext{
${ }^{1}$ Doctor en Teología-Pontificia, Università Lateranense, Italia. Profesor Asociado en la Carrera de Educación Física de la Universidad Nacional de la Matanza (UNLaM).

Correo electrónico: pbzanor@gmail.com
} 
with a beautiful body. Finally, we will try to propose the importance of reliving physical activity from the Hippocratic point of view to give it back its educational mission. This does not mean neglecting the aesthetic dimensions that are also part of physical activity.

Keywords: physical activity, Hippocratic theory, tyranny of beauty, identity of the person.

\section{La actividad física en la concepción Hipocrática}

El siguiente ensayo se ubican dentro del proyecto de investigación titulado: Análisis de la teoría hipocrática para entender el lugar de la actividad física en las sociedades del siglo XXI Su director es el Dr. Walter Toscano y la misma se realiza dentro del ámbito de investigación de la Universidad Nacional de la Matanza.

Como señala Toscano, podemos abordar a Hipócrates desde dos ópticas, como autor de la realización de una hazaña médica y como parte de la cultura griega (Toscano, 2008, p.69). En efecto, no debemos perder de vista que todo el pensamiento griego se contextualiza dentro del concepto de Paideia, a diferencia de hoy que cada área del saber aborda un ámbito humano sin relación al todo. Este hecho es fundamental para evita anacronismos que nos lleven a una lectura de la actividad física hipocrática con categorías modernas que terminarían desvirtuando su significado. Por otra parte, leer la actividad física como la plantea Hipócrates, y dentro de su contexto adecuado. nos permitirá repensar su significado para el hombre moderno.

\subsection{La paideia griega y la areté}

El ideal educativo de la antigua Grecia se configuró en la búsqueda de la armonía entre la educación física, la educación intelectual y la educación moral. La paideia incluye un proceso de crecimiento y de maduración en donde se aprenden las virtudes éticas, el acondicionamiento físico y las respuestas prácticas para la vida cotidiana.

La paideia es la denominación acuñada por Isócrates, uno de los pedagogos más representativos de la antigua Atenas. Educador que presentó luces claras sobre el concepto y la metodología de este estilo educativo, bien organizado y rico en cuestiones filosóficas. Es así como se ve representada en los diferentes escritos de Platón (Duarte-Osorio, 2020, p.374).

Los ciudadanos educados por la paideia aprendían música y gimnasia, filosofía y matemáticas, física y astronomía, y cualidades necesarias para la vida en la ciudad. El ejercicio del cuerpo, sumado al cultivo de la virtud del alma, podrían en resumen decirse que eran el principal objetivo de la paideia. En la Grecia antigua este concepto representaba la formación integral mediante la cual los individuos eran estructurados en los principios, en los 
valores fundamentales y con sólidos conocimientos para enfrentar los retos de la vida (Duarte-Osorio, 2020,).

Por otra parte, los filósofos, quienes en esa época se encontraban en uno de los momentos más lúcidos de la historia, construyeron las diferentes teorías naturalistas que sirven a la medicina como sustento para las hipótesis sobre la etiología de las enfermedades, el estudio sobre las estaciones y la dietética (Duarte-Osorio, 2020).

Por otra parte, esta formación integral de la persona era misión de la sociedad que se desarrollaba a través de la educación de las virtudes, el areté. Este último implicaba el desarrollo de las virtudes de la valentía, (andreia), de la moderación (sofrosine), de la justicia (diacaiosine) y de la prudencia (frónesis), para poder vivir en sociedad. Implicaba también la formación cultural, la música, la poesía, la oratoria y la retórica. Finalmente, la formación física mediante el dominio del cuerpo a través de la gimnasia y el deporte en general, los ejercicios físicos y el manejo de las armas. La gimnasia se practicaba en la palestra terreno deportivo al aire libre, cuadrado y rodeado de muros. Los deportes más practicados eran la lucha, la carrera, el salto y el lanzamiento de disco y de jabalina y el pancracio.

Pero lo importante de todo esto es no olvidar que ni la gimnasia tenía como misión cultivar lo corporal, ni, en el caso de la música, cultivar lo espiritual, sino que toda la educación apuntaba a la educación del hombre integral, entendido como un todo (Cachón Zagala et al. 2013).

En este sentido es que podemos hablar de una sociedad orgánica y ética. Orgánica en cuanto cada ámbito social, si bien poseía su propia autonomía, apuntaba a un mismo fin: la formación de la persona, y es este sentido que hablamos de una sociedad ética.

\subsection{Actividad física y areté en algunos autores}

Antes de llegar a Grecia, en la evolución del hombre, la actividad física ha jugado un papel fundamental y se ha adaptado a las necesidades humanas y de su entorno, siendo la base de su supervivencia cuando la utilizaba para desplazarse, cazar, pescar o defenderse; hasta llegar a formar parte de la educación, hecho que acontece por primera vez en la Grecia Clásica donde, además de formar parte de la preparación bélica, también integró la educación de los jóvenes y se convirtió en la base de los juegos (especialmente a través de los Juegos Olímpicos) e hizo sus primeras incursiones sistematizadas con la salud (Cachón Zagala et al. 2013).

Para Platón, quienes estuvieran llamados a solucionar grandes problemas de pensamientos, deberían practicar, al mismo tiempo, la gimnasia, siempre ejercitando, conjuntamente, el cuerpo y el alma. En La Academia, donde impartía sus clases Platón, a la 
vez que se instruía en las ciencias, la cultura, la política y las relaciones sociales, se dedicaba un gran tiempo a la actividad física.

Aristóteles incluye el tratamiento del cuerpo en la educación con un sentido médicohigienista, alejándose de las prácticas "gymnicas", ya profesionales, a las que considera excesivas y poco saludables.

Para Galeno, en tanto, la actividad física debía producir gozo y deleite en el alma, puesto que poseía todas las potencialidades del ser humano (Cachón Zagala et al. 2013).

\subsection{Actividad física y areté en Hipócrates}

Casi todos los filósofos y médicos griegos se han ocupado del atletismo, pero más que ningún otro lo hizo el padre de la medicina: Hipócrates. Él se ocupó de los ejercicios y del entrenamiento de los atletas, del régimen que debían seguir, prescribiendo el tipo de alimentación a ingerir para aumentar sus fuerzas, e indicando -al mismo tiempo- la manera de combatir su sed. Además, recomendaba el masaje y un pequeño paseo después de los ejercicios como rutinas saludables (Toscano, 2008).

Hipócrates insistió en que el exceso de ejercicio resultaba perjudicial, y que era difícil fijar y medir la cantidad de ejercicio conveniente a cada persona. Reconoció, también, la importancia de los movimientos como factor de la eliminación de los productos inútiles o perjudiciales para el cuerpo humano. Estudió las fatigas y sus causas, al mismo tiempo que su profilaxis y su tratamiento, prescribiendo el masaje y la hidroterapia (Toscano, 2008).

Uno de los grandes aportes del corpus Hipocrático es el concepto de Dieta, es decir la relación entre actividad física (gimnasia y ejercicios), el régimen y la salud. De este modo "la medicina hipocrática consistirá en una dietética, la cual ha de estar conformada por una ciencia de regímenes y una gimnasia o ciencia de la eficacia de los ejercicios" (Toscano, 2008, p.73). Es lo que hoy conocemos como entrenamiento deportivo. Pero, en palabras de Toscano (2007): "la dieta hipocrática no tiene nada que ver con la dieta atlética de nuestros tiempos, centrada en el alto rendimiento" (Toscano, 2007, p.140)

Acerca de la dieta dice Hipócrates: "Si además de esto pudiera hallarse en cada caso la proporción de alimentos y el número adecuado de ejercicios que no ofrecieran un desequilibrio ni por exceso ni por defecto, se descubriría con exactitud la salud para los hombres" (Toscano, 2008, p.75). Este brevísimo resumen del concepto de dieta en el corpus hipocrático, lo debemos ver como una parte del ideal educativo de la época que, a través del areté, no solo incluía la fuerza y la destreza, sino también la nobleza, la prudencia y la astucia (Toscano, 2007). En otras palabras, todo el pensamiento debe ser contextualizado dentro del marco de la paideia. 


\section{La actividad física en la visión estética de la vida contemporánea}

Mientras en la sociedad griega la visión del hombre y de la vida se encuadraban dentro del marco de la Paidea, es decir dentro de una sociedad orgánica en donde cada una de sus partes apuntaban a un mismo fin; para la excelencia de la persona, en la sociedad moderna, esto no ocurre así.

Podemos definir a la sociedad moderna como una sociedad compleja, es decir, en donde cada una de sus partes es autónoma e incluso compite con los otros ámbitos de la vida. Se perdió un ideal de vida buena como principio rector de las actividades sociales. El fin último del vivir en sociedad es relegado al ámbito privado, y el sujeto moderno encuentra mucha dificultad para poder interpretar, o descubrir, su misión y su lugar en la sociedad. Es más, se produce una tensión entre persona y sociedad. Debido a esto, al sujeto moderno le cuesta mucho valorar lo que hace.

Este hecho es constatado por los estudios de calidad de vida que indican un desencanto del hombre moderno en relación a su ser y a su vivir en sociedad. La paradoja de Easterlin puso de relieve, precisamente, el descontento de las personas en las sociedades modernas opulentas. "Si a esto le sumamos una perspectiva psicológica, no solo la felicidad de los países no se ha incrementado, sino que sí observamos la tasa de prevalencia de algunos trastornos psicológicos" (Castro Solano y Tonon, 2013, p.220)

Al no existir un principio unificador que pueda articular los distintos ámbitos de la vida, la persona se ve tironeada por cada uno de ellos al punto de desgarrarse existencialmente. Dentro de este contexto, las expectativas de las personas tienden a buscar aquellas realidades y actividades que puedan gratificarlas; y como las experiencias sensibles producen una gratificación rápida y segura, la vida de las personas se termina por reducir a la búsqueda de ellas, es decir: una buena comida, un celular, un viaje, indumentaria, una buena película y otros bienes sensibles.

En otras palabras, el problema del vivir ya no es más un problema ético, es decir, de búsqueda de la excelencia de la persona (areté), sino que se enfoca en sentirse bien y, por lo tanto, queda reducido a un problema clínico. En palabras de Tonon (2013): "Si bien Aristóteles ligó la felicidad al ejercicio de la virtud, en la sociedad actual, no es esta versión de la felicidad la que predomina sino una versión más de corte hedonista ligada a la acumulación de placeres y al incremento de emociones positivas" (p. 220).

Dentro de este contexto, no es difícil advertir la importancia que asume lo estético y la imagen (en especial las redes sociales) sobre los valores de la persona, al punto de constituirse en una verdadera tiranía de la belleza y un culto al cuerpo. La obsesión por el 
cuerpo perfecto, por las apariencias lleva a muchas personas a sufrir y a padecer sudor y lágrimas con tal de lograr ser bellos (Gervilla Castillo, 2003, p.187).

\subsection{El culto al cuerpo bello}

El culto a la belleza del cuerpo actual condiciona el éxito, las amistades, las posibilidades laborales y en definitiva la aceptación social. De este modo, se subordina el descanso, el vestido, el alimento, y gran parte del dinero, al aspecto físico. Tener un físico agradable se ha convertido en una «obligación», pues ello supone prestigio, seguridad y superioridad, aunque, a veces, se este se alcance a costa del sacrificio de otros valores más humanizantes. Hoy el narcisismo ha quedado asociado a la apariencia corporal, a la belleza, a la satisfacción personal, al triunfo, y a la autoestima. Somos voraces consumidores del cuerpo delgado, joven y sensual. Nunca el cuerpo ha influido tanto en el yo, y en el autoconcepto. No se trata del cuerpo en su totalidad, sino de su apariencia física, silueta, talla, color, tal como exhiben los modelos encarnados en las personas sobrevaloradas socialmente: deportistas, famosos, top-models, actores y actrices.

Y cuando el propio cuerpo no está acorde con los modelos vigentes en la sociedad, se trata de corregir la obra de la naturaleza, de vencer los estragos ocasionados por el paso del tiempo, de sustituir el cuerpo recibido por un cuerpo construido (Gervilla Castillo, 2003).

De este modo se plantea una oposición entre educación integral (paideia) y la tiranía de la belleza (Gervilla Castillo, 2003). Se ha cedido el valor del cuerpo a la estética, y lo justo al gusto. La búsqueda de la perfección moral ha sido sustituida por la perfección corporal.

Este culto al cuerpo ha originado todo un mercado de negocios, cosméticos, cremas, dermo-protectores, de los gimnasios, pistas de squash y paddle para ejecutivos, aerobic, maratones, productos dietéticos, revistas, herbolarios.... De este modo, la actividad física se contextualiza dentro de un marco de referencia totalmente distinto al de la Antigüedad, desenganchada de la persona misma, y relacionada frecuentemente con las dimensiones estéticas de un cuerpo bello y esbelto.

\subsection{La actividad física y la belleza en la actualidad}

La tiranía de la belleza corporal resignifica el sentido de la actividad física. El deporte, en tanto práctica del sujeto, es considerado, muchas veces, como una herramienta modeladora del cuerpo, sin principios ni valores, en donde todo vale con tal de lograr el cuerpo vigoréxico en los varones, y excesivamente delgado en las mujeres.

El uso de esteroides, la práctica de entrenamientos contraindicados, o las rutinas extenuantes, son parte del escenario que muchas veces acompaña la actividad física contemporánea. Así, los ejercicios que se practican buscan modelar los brazos, el abdomen, las piernas y la cadera (Hormiga Sánchez et al. 2019). 
Por otra parte, en los adolescentes varones, el estereotipo clásico facilita que experimenten una mayor presión social hacia un ejercicio físico que propicie una imagen de fuerza y potencia, mientras que los modelos femeninos alientan una actividad física dirigida a la consecución de una imagen corporal que mezcle un cuerpo delgado (ectomórfico) con un cuerpo más musculoso, unas caderas y unos músculos más pequeños y fuertes; al tiempo que: hombros más anchos y brazos con una musculatura perfilada (Fernandez et al. 1999).

A similar conclusión arriba el estudio realizado por Mieziene et al (2014) cuando señalan que la actividad física intensa y moderada practicada por los adolescentes, no está asociada a un estilo de vida saludable.

\section{La identidad de la persona humana y la actividad física}

El cuerpo, entendido como corporeidad, es decir, como expresión de la subjetividad de una persona, supone el movimiento del cuerpo y, por lo tanto, la actividad física, como la condición de posibilidad para que la persona pueda desarrollar su identidad.

En este punto es donde se encuentra la labor educativa de los docentes de las distintas áreas, incluidos los profesores de educación física. En este sentido, el ideal educativo clásico integraba de forma armónica la formación intelectual, musical y física de la persona.

En el nuevo paradigma moderno, al desentenderse de la identidad de la persona y, en definitiva, al negar a la persona misma en cuanto tal, termina por asociar la actividad física a lo bello y estético según parámetros sociales aceptados, y a su vez promovidos. por los medios de comunicación. De este modo, persona/identidad y actividad física se separan aumentando el nivel de insatisfacción de la persona.

\section{Conclusión}

La paideia griega se elevaba de lo particular a lo universal, de la belleza sensible a lo bello universal. La modernidad quiebra esta relación de modo tal que ya no existe lo bello sino algo bello. "La belleza ha muerto y de sus cenizas han nacido las bellezas, el politeísmo estético" (Gervilla Castillo, 2003, p.197)

La belleza moderna se impone como tiránica y excluyente de otros valores como la salud, el descanso y la buena alimentación, esto supone un predominio de lo estético sobre lo ético. No se trata de negar el valor de la estética, sino de afirmar que esta solo es valiosa si antes se vive y se respeta la ética. Lo que desvaloriza la estética hasta volverla odiosa, es 
el carecer de una base ética, es decir, el hecho de impedir el desarrollo integral y la identidad de la persona (Gervilla Castillo, 2003).

Dentro de este marco se sitúa gran parte de la actividad física realizada por muchas personas que se someten a grandes esfuerzos, dietas que muchas veces perjudican la salud con tal de poseer un cuerpo bello. Es sabido como aumenta la concurrencia a los gimnasios con el comienzo de la primavera y la preocupación por llegar al verano con un cuerpo bien parecido.

La actividad física griega, y en particular la Hipocrática, nos desafía hoy a volver a integrar lo bello y lo bueno. En otras palabras, entender que la actividad física no solo modela el cuerpo, sino también el espíritu de la persona y su identidad misma. También nos ayuda a vincular la actividad física con los valores y, en especial, con aquellos asociados a la fraternidad, la perseverancia, la solidaridad, la paz y la magnanimidad.

Tendríamos que intentar vivir la actividad física como una gimnasia del cuerpo, pero también del espíritu, es decir, como una escuela de vida (Sánchez Pato y Se la Torre Olid, 2016).

\section{Referencias bibliográficas}

Cachón Zagalaz, J., Castro López, R., Valdivia, P. y Zurita Ortega, F. (2013). Actividad física y educación en la Grecia clásica I. Trances, 5(3), 271-280.

Castro Solano, A. y Tonon, G. H. (2013). Naciones Felices: Mas allá del Dinero. Estudos Contemporâneos da Subjetividade, 3(2), 219-228. http://www.periodicoshumanas.uff.br/ecos/article/view/1252

Duarte Osorio, L. (2020). La formación médica y el concepto "paideia”. Revista Ciencias Biomédicas, 3(2), 373-376. https://doi.org/10.32997/rcb-2012-3137

Fernández M. J, Juan J., Marcó Arbonés M., Gracia Blanco, M. (1999). Autoconcepto físico, modelo estético e imagen corporal en una muestra de adolescentes. Psiquis: Revista de psiquiatría, psicología médica y psicosomática, 20(1), 27-38.

Gervilla Castillo, E. (2003). La tiranía de la belleza, un problema educativo hoy. La estética del cuerpo como valor y como problema. Teoría de la educación. Revista Interuniversitaria, 14, 185-206. https://doi.org/10.14201/2990

Hormiga Sánchez, C. M., Alzate Posada, M. L. y Cortés-García, C. M. (2019). Significados de la actividad física en la cotidianidad. Los lugares de la belleza y el placer en 
una práctica de salud. Revista Ciencias de la Salud, 17(spe), 12-31.

https://doi.org/10.12804/revistas.urosario.edu.co/revsalud/a.8110

Mieziene, B., Jankauskiene, R. y Mickunieneb, R (2014). Can Internalization of Sociocultural Beauty Standards Predict Adolescents' Physical Activity? Procedia - Social and Behavioral Sciences, (116). 956-961.

https://doi.org/10.1016/j.sbspro.2014.01.327

Sánchez Pato, A. y de la Torre Olid, F. (2016). Reflexiones para una educación éticoestética de la belleza como contribución a la dignificación de la persona en el deporte. RECERCA. Revista De Pensament I Anàlisi, (18), 86-109. https://doi.org/10.6035/Recerca.2016.18.6

Toscano W. (2007). Los ejercicios físicos y la calidad de vida en el corpus hipocrático. Hologramática, año IV, 1(7),127-144.

Toscano, W. (2008) Los ejercicios físicos y la salud en el corpus Hipocrático. Calidad De Vida y Salud, 1(1),67-

82. http://revistacdvs.uflo.edu.ar/index.php/CdVUFLO/article/view/6 